\title{
Determination of Preload of Double-Row Tapered Roller Bearing Used for Supporting Direct-Drive Wind Turbine Rotor
}

\author{
Yunfeng $\mathrm{Li}^{1}$ \\ ${ }^{1}$ School of Mechatronical Engineering, Henan University of Science and Technology, Luoyang, China \\ Correspondence: Yunfeng Li, 66\#, Xiyan Campus, Henan University of Science and Technology, 48 Xiyan Road, \\ Luoyang 471003, China. Tel: 86-134-6108-0135. E-mail: liyunfeng379@126.com
}

Received: November 25, 2013

Accepted: December 13, 2013 Online Published: December 21, 2013

doi:10.5539/mer.v4n1p27

URL: http://dx.doi.org/10.5539/mer.v4n1p27

\begin{abstract}
Appropriate axial preload is necessary for the double-row tapered roller bearing used for supporting the rotor of the direct-drive wind turbine; its function is to ensure the rolling motions of the rollers and the long fatigue life of the bearing as far as possible. For this purpose, statics model of the preloaded bearing under the combined action of radial load, axial load and tilting moment load was established firstly; then, for a set of selected preload values which are different proportions of the dynamic equivalent axial load of the external bearing loads, the corresponding loaded roller number, maximum roller load and bearing fatigue life were obtained; thirdly, the effects of different preloads on the calculated indicator values were analyzed, result show that preload can improve the uneven load distribution among the rollers, the preload value also influence the rolling roller number and bearing fatigue life. A preload of 0.5 times of the dynamic equivalent axial load was selected as a trade-off between the rolling roller number and bearing fatigue life.
\end{abstract}

Keywords: wind turbine, double-row tapered roller bearing, statics analysis, axial preload, rolling motion, fatigue life

\section{Introduction}

In spite of the global economic depression, the worldwide wind capacity reached $254000 \mathrm{MW}$ by the end of June 2012, out of which 16546 MW were added in the first six months of 2012 (World Wind Energy Association, 2012). The reliability of wind turbines has attract more and more attention from the researcher, bearings are the key components of wind turbines, they need to be pay more attention as they have higher costs associated with repair or replacement (Kotzalas \& Doll, 2010). In recent years, some research work of the mechanics problems of slewing bearing used for wind turbine have been conducted by the researchers. Such as the calculation of the static load-carrying capacity of four-contact-point slewing bearings used for the pitching system and yawing system of wind turbine (Aguirrebeitia, Plaza, Abasolo, \& Vallejo, 2013), the contact fatigue damage of hardened layer of bearing raceway used for the yaw system in wind turbine, this problem was solved by constructing the nonlinear material constitutive equation of hardened layer (Niu, Yang, \& Gao, 2013), and the effect of local structure change on the fatigue life of yaw slewing bearing of wind turbine, this problem was analyzed by the FEM software (Feng, Chen, Huang, \& Wang, 2013), all the present work mainly aimed at the slewing bearings used for the pitching system and yawing system of the double-fed induction wind turbine.

Direct-drive wind turbine is a new turbine architecture which has been developed in recent years, this kind of wind turbine eliminate the gearbox and connect the rotor directly to a permanent magnet generator. Direct-drive wind turbine offers significant potential because they eliminate the gear-speed increaser, which is susceptible to significant accumulated fatigue torque loading, related reliability issues, and maintenance costs (Bywaters et al., 2007).

The rotor of direct-drive wind turbine is supported by one set of slewing bearing. Double-row tapered roller bearing is deemed as a more suitable selection to support the rotor of direct-drive wind turbine (Lucas \& Pontius, 2005), because this kind of bearing can carry the combined actions of radial load, axial load and tilting moment load; and their design is built around the concept of zero slip which minimizes wear over long periods of operation; especially their load distribution among the rollers can be optimized to avoid load losing or exceeding of some rollers through appropriate axial preload. The cross section of double-row tapered roller bearing is shown in Figure1. In the direct-drive wind turbine, the outer ring of the bearing is connected to the nacelle main 
frame, and the inner ring of the bearing is connected to the rotor. During the operating process, the bearing sustains the axial load which comes from the aerodynamic load from the rotor, the tilting moment load which comes from the aerodynamic load from the rotor and the weight of the rotor, the radial load form the weight of the rotor mainly.

Axial preload is needed by double-row tapered roller bearing to make the load be well distributed among the two row rollers, its aim is to ensure nearly all rollers to carry load and roll autonomously. As the preload is insufficient, the action of external axial load will result in the unloading of some rollers. Because the normal contact loads between the unloaded rollers and the raceway equal to zero, the raceway surface of the rotating ring can not drag the unloaded rollers to orbit through rolling motion by frictional traction. Slipping frictions appear between the unloaded rollers and the raceway surface; friction heat will lead to the premature failure of the raceway and the cage. While as the preload is excessively large, the combining action of the preload and the external load can result in the exceeding of the roller loads over the rated load, and this will result in heat generation and fatigue failure of the bearing. Therefore, it is necessary to understand deeply the influence laws of preload on the load distribution of the bearing rollers and determine the reasonable value of the preload based on these laws further.

The intend of this paper is to establish the static model of axial preloaded double-row tapered roller bearing used for supporting the rotor of direct-drive wind turbine, and analyze the effect of axial preload on the loaded roller number, maximum roller load and bearing fatigue life; use these analyzed results to determine the appropriate axial preload.

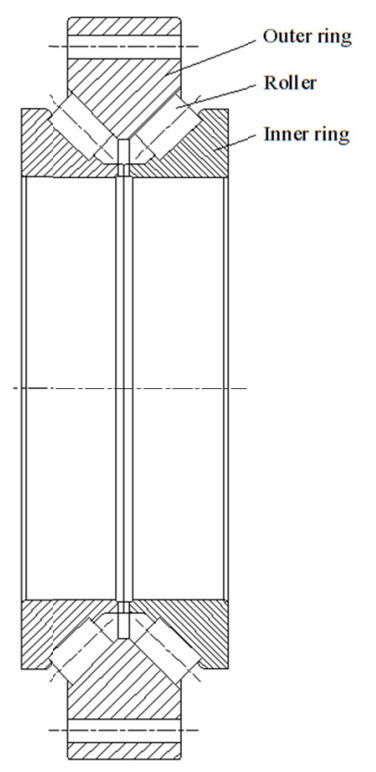

Figure 1. Double-row tapered roller bearing

\section{Force Analysis for Tapered Roller}

In the tapered roller bearings, in addition to bear the normal loads from the inner raceway and outer raceway, the tapered roller also bear the normal loads from the guide flange of the inner ring. Suppose the acting force between the roller and the inner raceway, outer raceway, guide flange is $Q_{i}, Q_{e}, Q_{f}$ respectively; and the corresponding contact angle is $\alpha_{i}, \alpha_{e}, \alpha_{f}$ respectively, as shown in Figure 2. 


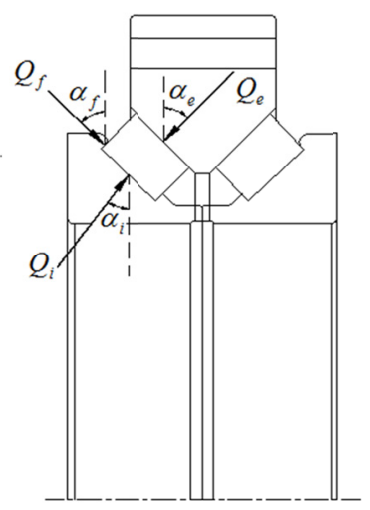

Figure 2. Forces of tapered roller

For the static equilibrium of the tapered roller, the sum of forces in any direction is equal to zero, therefore,

$$
\begin{aligned}
& Q_{e} \sin \alpha_{e}-Q_{i} \sin \alpha_{i}-Q_{f} \sin \alpha_{f}=0 \\
& Q_{e} \cos \alpha_{e}-Q_{i} \cos \alpha_{i}+Q_{f} \cos \alpha_{f}=0
\end{aligned}
$$

For the systems of equations composed by Equation (1) and Equation (2), suppose $Q_{e}, \alpha_{i}, \alpha_{e}$ and $\alpha_{f}$ are known variables, $Q_{i}$ and $Q_{f}$ are unknown variables. Then $Q_{i}$ and $Q_{f}$ can be represented by other variables through solving the systems of equations, that is

$$
\begin{gathered}
Q_{i}=\frac{\sin \left(\alpha_{e}+\alpha_{f}\right)}{\sin \left(\alpha_{i}+\alpha_{f}\right)} Q_{e} \\
Q_{f}=\frac{\sin \left(\alpha_{e}-\alpha_{f}\right)}{\sin \left(\alpha_{i}+\alpha_{f}\right)} Q_{e}
\end{gathered}
$$

Between the roller and the outer raceway, only normal force $Q_{e}$ exists, so in the statics analysis of tapered roller bearings, the rollers and the inner ring are looked as an isolated body to consider its equilibrium problem. The rollers and the inner ring together are called "roller-inner ring" isolated body in the following.

\section{Load-Deflection Relationship}

When relative displacement between the inner ring and the outer ring emerges, because the contact angles of the inner raceway and the outer raceway are different, the elastic contact deformation between the roller and the inner raceway is not in the same direction as it between the roller and the outer raceway.

Here, the elastic contact deformation direction between the roller and the outer raceway is looked as a benchmark, project the elastic contact deformation direction between the roller and the inner raceway onto the elastic contact deformation direction between the roller and the outer raceway; the total approach between two raceways under load separated by a roller is the sum of the elastic contact deformations between the roller and each raceway. In this direction, the total load-deflection factor is (Lundberg \& Palmgren, 1947)

$$
K_{n e}=8.06 \times 10^{4} l^{0.89}\left[1+c_{i}^{0.9} \cos \left(\alpha_{e}-\alpha_{i}\right)\right]^{-1.11}
$$

in which

$$
c_{i}=\sin \left(\alpha_{e}+\alpha_{f}\right) / \sin \left(\alpha_{i}+\alpha_{f}\right)
$$

Then, the normal contact load between the roller and the outer raceway is

$$
Q_{e}=K_{n e} \delta_{n}^{1.11}
$$

\section{Equilibrium Model of Bearing Under Axial Preload}

After the bearing is mounted on the host machine, the axial preload is applied on the inner ring. As the bearing being analyzed, suppose the outer ring of the bearing is fixed. For the convenience of analysis, subscript "1" is used to represent the row adjacent to the rotor, and subscript " 2 " represent the row adjacent to the nacelle. 
The axial displacement between the rotor side row inner ring and the outer ring is $\delta_{a 0}$, the elasticity contact deformation along the normal direction of outer raceway produced by this axial displacement is

$$
\delta_{n e}^{0}=\delta_{a 0} \sin \alpha_{e}
$$

Substituting Equation (7) into Equation (6), the contact load $Q_{e}$ between the roller and the outer raceway can be obtained. Then the equilibrium equation of the rotor side row "roller - inner ring" isolated body is

$$
F_{a 0}-Z Q_{e} \sin \alpha_{e}=0
$$

Where, $Z$ is the roller number of each row. From Equation (8), one can obtain the axial displacement of the rotor side inner ring relative to the outer ring under the action of axial preload as follows:

$$
\delta_{a 0}=\left(\frac{F_{a 0}}{Z K_{n e}\left(\sin \alpha_{e}\right)^{2.11}}\right)^{0.9}
$$

Similarly, the axial displacement between the nacelle side row inner and the outer ring is $\delta_{a 0}$ too under the action of axial preload $F_{a 0}$.

\section{Equilibrium Model of Bearing Under Combined Loads}

Suppose the outer ring of the bearing is fixed, under the combined action of radial load $F_{r}$, axial load $F_{a}$ and tilting moment load $M$, the corresponding radial displacement, axial displacement and angular displacement of the bearing inner ring is $\delta_{r}, \delta_{a}$ and $\theta$ respectively. The "roller-inner ring" isolated body sustains the action of external loads $F_{r}, F_{a}$ and $M$, it also sustains the normal loads $Q_{e}$ acting on the roller from the outer raceway, as shown in Figure 3. Under the actions of these loads, the "roller-inner ring" isolated body is in an equilibrium state.

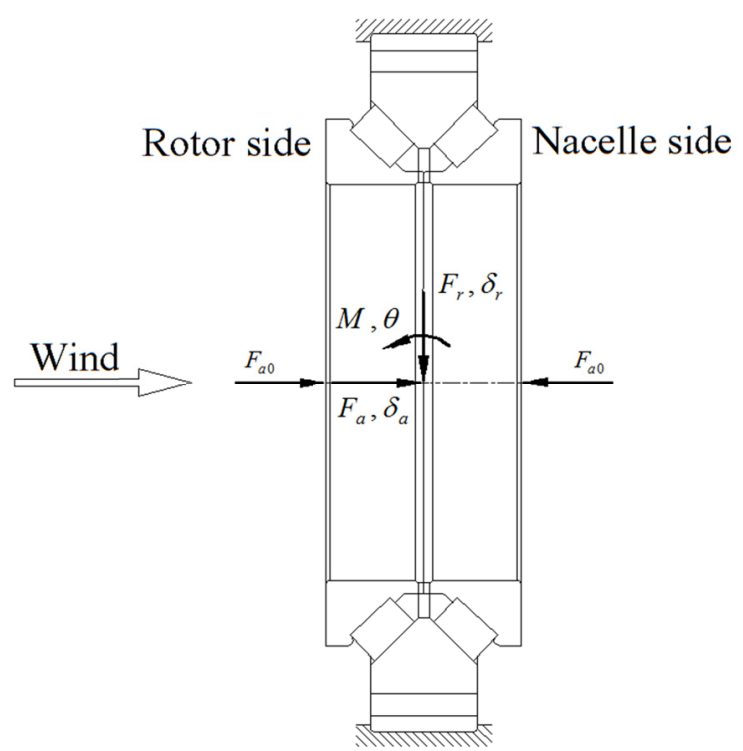

Figure 3. Displacements of inner ring under combined loads

\subsection{Displacement of Each Roller Position of Rotor Side Row}

Firstly, consider the rotor side row rollers, the axial displacement at the position of $j$ th roller produced by the axial displacement $\delta_{a}$ is equal to $\delta_{a}$, while the radial displacement at the position of $j$ th roller produced by the radial displacement $\delta_{r}$ is

$$
\delta_{r 1(j)}^{r}=\delta_{r} \cos \psi_{(j)}
$$

Where, $\psi_{(\mathrm{j})}$ is the position azimuth angle of the roller along the raceway. The radial displacement at the position of $j$ th roller produced by the tilting angular displacement $\theta$ is

$$
\delta_{r 1(j)}^{\theta}=0.5 d_{c} \theta \cos \psi(j)
$$


Where, $d_{c}$ is the axial distance between two row roller centers. The axial displacement at the position of $j$ th roller produced by the tilting angular displacement $\theta$ is

$$
\delta_{a 1(j)}^{\theta}=0.5 d_{m} \theta \cos \psi_{(j)}
$$

Where, $d_{m}$ is the pitch diameter of the bearing. The radial displacement at the position of each roller is produced by the radial displacement $\delta_{r}$ and the tilting angular displacement $\theta$. According to Equation (10) and Equation (11), the total radial displacement at the position of $j$ th roller is

$$
\delta_{r 1(j)}=\left(\delta_{r}+0.5 d_{c} \theta\right) \cos \psi_{(j)}
$$

For the axial displacement at the position of each roller, in addition to the axial displacement produced by the axial displacement $\delta_{a}$ and the tilting angular displacement $\theta$, the axial displacement produced by the axial displacement $\delta_{a 0}$ should be also considered. Then, the total axial displacement at the position of $j$ th roller is

$$
\delta_{a 1(j)}=\delta_{a 0}+\delta_{a}+0.5 d_{m} \theta \cos \psi_{(j)}
$$

Then, the total displacement at the position of $j$ th roller along the normal direction of the outer raceway is

$$
\delta_{n 1(j)}=\delta_{r 1(j)} \cos \alpha_{e}+\delta_{a 1(j)} \sin \alpha_{e}
$$

\subsection{Displacement of Each Roller Position of Nacelle Side Row}

According to the same principle as above, for the nacelle side row rollers, the axial displacement at the position of $j$ th roller produced by the axial displacement $\delta_{a}$ is equal to $-\delta_{a}$.

The total radial displacement at the position of $j$ th roller is

$$
\delta_{r 2(j)}=\left(\delta_{r}-0.5 d_{c} \theta\right) \cos \psi_{(j)}
$$

Similarly, the total axial displacement at the position of $j$ th roller is

$$
\delta_{a 2(j)}=\delta_{a 0}-\delta_{a}-0.5 d_{m} \theta \cos \psi_{(j)}
$$

The total displacement at the position of $j$ th roller along the normal direction of the outer raceway contact is

$$
\delta_{n 2(j)}=\delta_{r 2(j)} \cos \alpha_{e}+\delta_{a 2(j)} \sin \alpha_{e}
$$

\subsection{Equilibrium Equations of "Roller-Inner Ring" Isolated Body}

Substituting Equation (15) and Equation (18) into Equation (6) respectively, the expressions of normal load $Q_{e l(j)}$ and $Q_{e 2(j)}$ between the two row rollers and the outer raceway can be obtained, where, $j=1,2,3, \ldots, Z$.

In the radial direction of the bearing, the equilibrium equation of the "roller-inner ring" isolated body under the actions of external radial load $F_{r}$ and normal $Q_{e l(j)}$ and $Q_{e 2(j)}$ is

$$
F_{r}-\sum_{j=1}^{Z}\left(Q_{e 1(j)}+Q_{e 2(j)}\right) \cos \alpha_{e} \cos \psi_{(j)}=0
$$

In the axial direction of the bearing, the equilibrium equation of the "roller - inner ring" isolated body under the actions of external axial load $F_{a}$ and normal $Q_{e l(j)}$ and $Q_{e 2(j)}$ is

$$
F_{a}-\sum_{j=1}^{Z}\left(Q_{e 1(j)}-Q_{e 2(j)}\right) \sin \alpha_{e}=0
$$

The normal load $Q_{e l(j)}$ acting on the rollers by the outer raceway will produce moment actions on the "roller-inner ring" isolated body. One part of them is the moment produced by the axial component of load $Q_{e l(j)}$ on the bearing center, another part of them is the moment produced by the radial component of load $Q_{e l(j)}$ on the bearing center. The equilibrium equation of the "roller-inner ring" isolated body under the actions of the two part moments and external tilting moment load $M$ is

$$
M-0.5\left(d_{m} \sin \alpha_{e}+d_{c} \cos \alpha_{e}\right)\left(\sum_{j=1}^{z} Q_{e 1(j)} \cos \psi_{(j)}-\sum_{j=1}^{z} Q_{e 2(j)} \cos \psi_{(j)}\right)=0
$$

A system of nonlinear equations can be obtained by using the above three equilibrium Equations (19)-(21). If the design parameters of the bearing are given, the values of unknown variable $\delta_{r}, \delta_{a}$ and $\theta$ can be solved corresponding a set of axial preload $F_{a 0}$ and external loads $F_{r}, F_{a}$ and $M$, and the roller loads can be calculated 
further.

\section{Example Calculation and Analysis}

The design parameters of the rotor bearing of certain type 2.0 MW direct drive wind turbine are listed as in Table 1 , and the external fatigue loads are $F_{r}=451(\mathrm{kN}), F_{a}=544(\mathrm{kN}), M=1072(\mathrm{kNm})$. According to T. Harris (Harris, Rumbarger, \& Butterfield, 2009), the dynamic equivalent axial load of the bearing can be calculated by using these external fatigue loads, the calculated value is as follows: $F_{a e}=1941(\mathrm{kN})$. In this paper, the value of $F_{a e}$ is used as a baseline for determining the axial preload of the bearing. As the axial preload equals to the different proportion of $F_{a e}$, the results calculated are shown in Figure 4-Figure 8.

Table 1. Bearing design parameters

\begin{tabular}{ccccccccc}
\hline$d_{m}(\mathrm{~mm})$ & $d_{c}(\mathrm{~mm})$ & $a_{i}\left({ }^{\circ}\right)$ & $a_{e}\left({ }^{\circ}\right)$ & $a_{f}\left({ }^{\circ}\right)$ & $l_{w e}(\mathrm{~mm})$ & $D_{w e}(\mathrm{~mm})$ & $\phi\left({ }^{\circ}\right)$ & $Z$ \\
\hline 2026 & 116 & 43 & 45 & 46 & 90 & 51 & 1 & 104 \\
\hline
\end{tabular}

The uneven distribution of roller loads can be attributed to the actions of external axial load and tilting moment load, axial load mainly make the uneven distribution between two rows. Uneven distribution of roller loads can result in the roller slipping, heat generation and fatigue life decrease. Axial preload can improve the uneven load distribution of the rollers. With the increase of axial preload, more and more rollers begin to undertake the bearing external loads, this can be seen in Figure 4 and Figure 5 . When the preload is less than $0.6 F_{a e}$, the normal contact loads between some rollers and the raceway are equal to zero, these rollers can not roll by themselves, their orbiting are realized by the push of the cage, this is disadvantageous to the bearing. When the preload is equal to $0.6 F_{a e}$, the normal contact loads of all rollers of the two rows are nonzero, they all can realize pure rolling, this is advantageous for the bearing to reduce friction and heat generation. When the axial preload is larger than $0.6 F_{a e}$, with the increase of the preload, the roller loads begin to increase distinctly; this is disadvantageous to the bearing; because excessive large roller load can result in the premature fatigue failure of the raceways. From the viewpoint of slipping friction and heat generation, the preload of $0.6 F_{a e}$ is most favorable for the bearing.

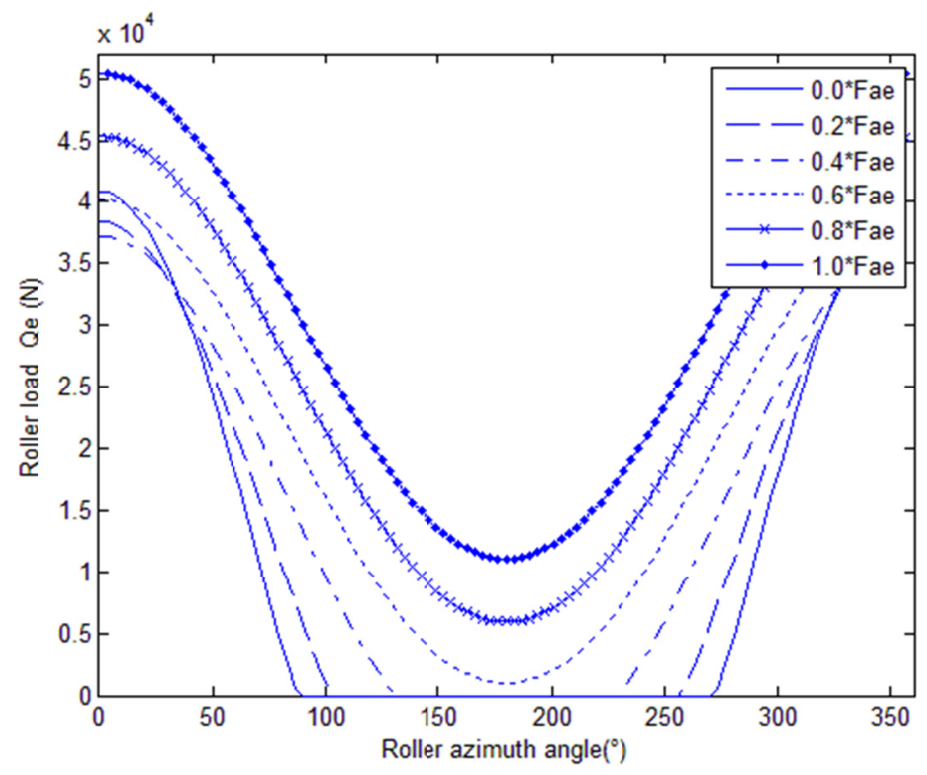

Figure 4. Load distribution of rotor side row 


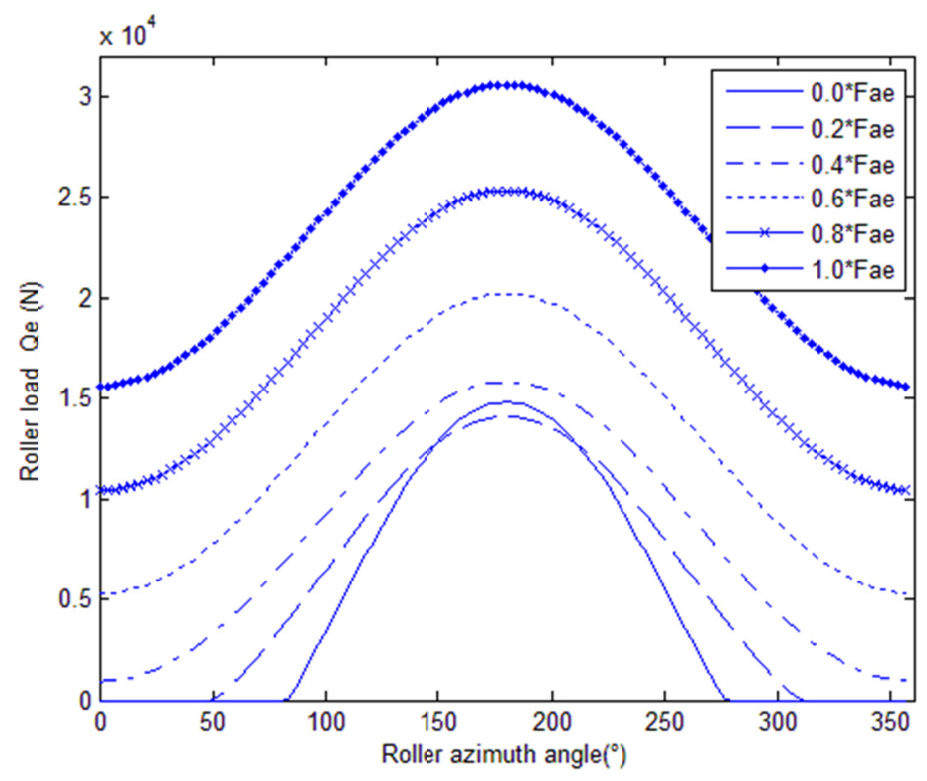

Figure 5. Load distribution of nacelle side row

The effect of the preload on the fatigue life of the bearing is shown in Figure 6. With the increase of the preload from 0 to $0.3 F_{a e}$, the bearing fatigue life increase accordingly; this can be attributed to the reason that the load distribution among the rollers become more and more even. When the preload is larger than $0.3 F_{a e}$, with the increase of the axial preload, the bearing fatigue life begins decrease distinctly; this can be attributed to the reason that the roller loads begin to increase due to the preload, as shown in Figure 7. Generally speaking, the effect of the roller load increase exceeds the distribution evenness increase through preloading. From the viewpoint of fatigue failure, the preload of $0.3 F_{a e}$ is most favorable for the bearing.

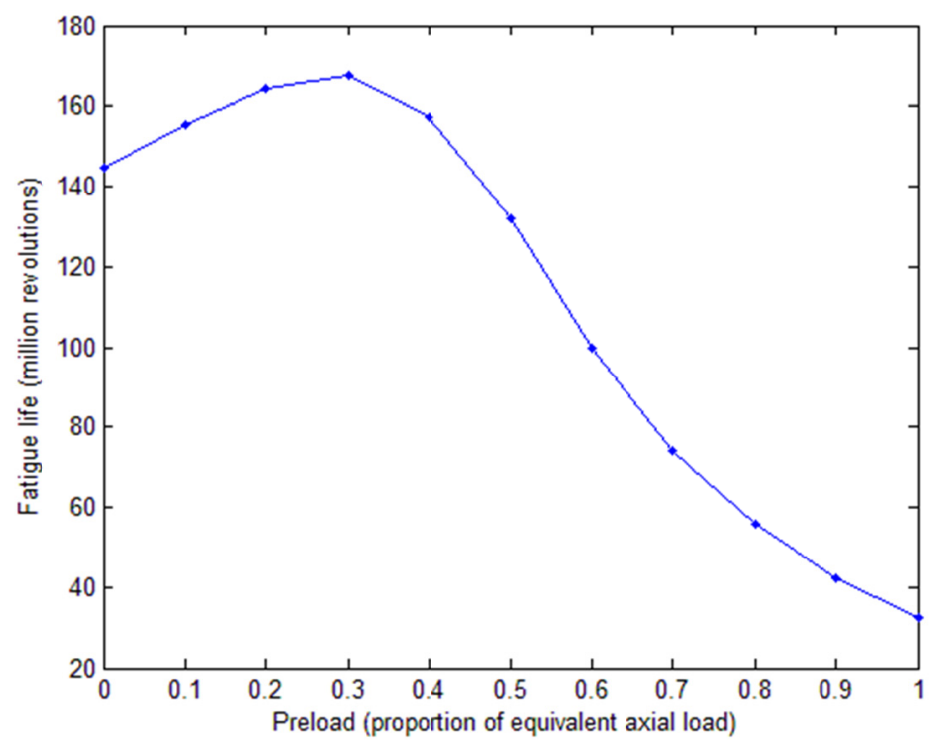

Figure 6. Bearing fatigue life 


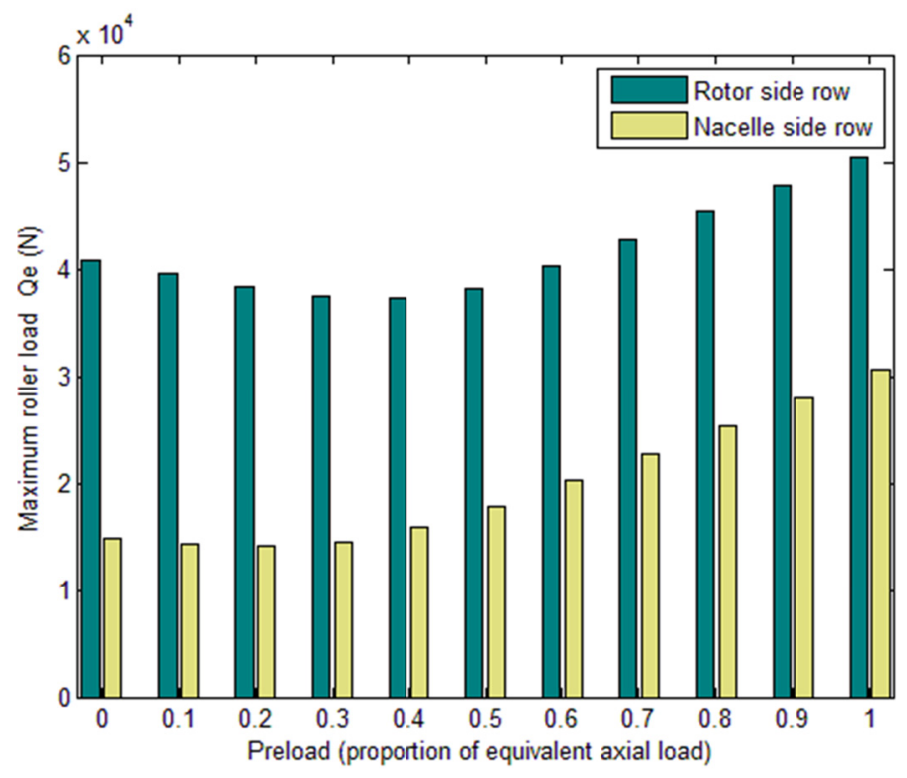

Figure 7. Maximum roller load

Determination of the preload should consider to reduce both roller slipping and raceway fatigue failure. For the former, the proportion of the loaded rollers should be no less than 80 per cent so as to over come the effect of slippage. According to Figure 8, the preload need to be larger than $0.4 F_{a e}$. For the latter, if the preload is larger than $0.5 F_{a e}$, fatigue life will decrease drastically, as shown in Figure 6 . Therefore, as a trade-off selection, $0.5 F_{a e}$ is selected as the bearing preload.

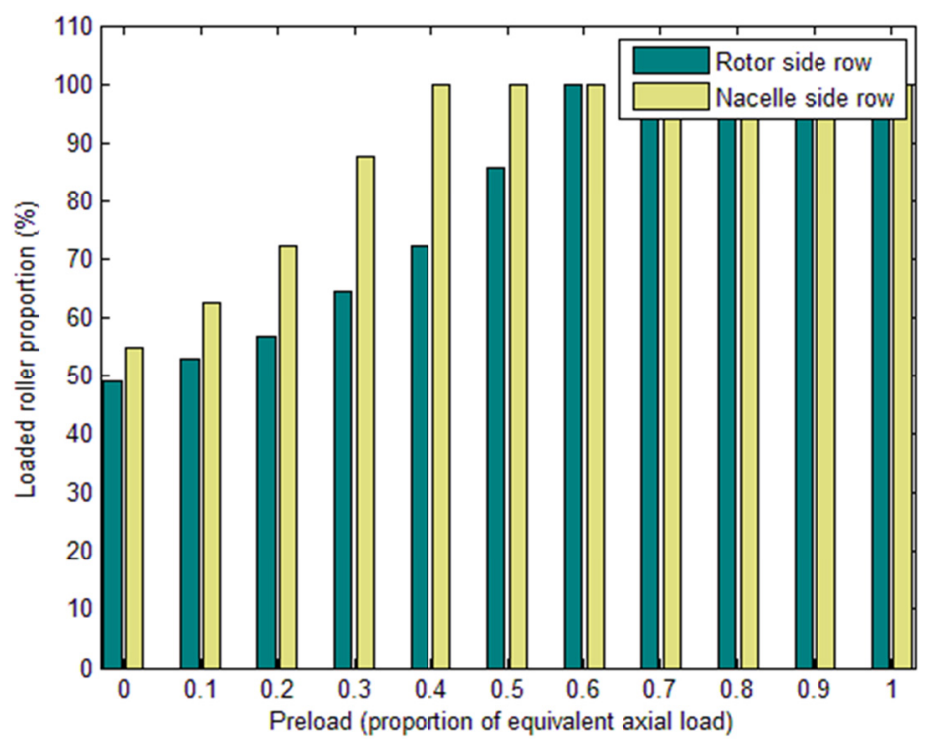

Figure 8. Proportion of loaded rollers

\section{Conclusion}

Double-row tapered roller bearing is a suitable selection to support the rotor of direct-drive wind turbine, and the appropriate preload is necessary for the bearing to ensure the rolling motions of the rollers and the long fatigue life of the bearing as far as possible. Statics model of the preloaded bearing was established, for a set of selected preload values which are different proportions of the dynamic equivalent axial load, the loaded roller number, maximum roller load and the bearing fatigue life were calculated. With the increase of the preload, the number of loaded rollers becomes more and more, this is advantageous for the pure rolling of the rollers; but excessive large preload will increase the roller loads and decrease the fatigue life of the bearing. Based on these results, a 
preload of 0.5 times of the dynamic equivalent axial load was determined.

\section{Acknowledgements}

The research is financed by the Key Projects of Science and Technology Research of the Education Department of Henan Province (Grant No.14A460008).

\section{References}

Aguirrebeitia, J., Plaza, J., Abasolo, M., \& Vallejo, J. (2013). General static load-carrying capacity of four-contact-point slewing bearings for wind turbine generator actuation systems. Wind Energy, 16(5), 759-774. http://dx.doi.org/10.1002/we. 1530

Bywaters, G., Mattila, P., Costin, D., Stowell, J., John, V., Hoskins, S., ... Freeman, B. (2007). Northern Power NW 1500 Direct-Drive Generator. US National Renewable Energy Laboratory, USA.

Feng, Y., Chen, J., Huang, X. D., \& Wang, W. (2013). Structure fatigue life analysis of a 1.5 MW smart yaw slewing bearing. Advanced Materials Research, 779, 664-670.

Harris, T. A., Rumbarger, J. H., \& Butterfield, C. P. (2009). Wind turbine design guide DG03: Yaw and pitch rolling bearing life. US National Renewable Energy Laboratory, USA. http://dx.doi.org/10.2172/969722

Kotzalas, M. N., \& Doll, G. L. (2010). Tribological advancements for reliable wind turbine performance. Phil. Trans. R. Soc. A, 368, 4829-4850. http://dx.doi.org/10.1098/rsta.2010.0194

Lucas, D., \& Pontius, T. (2005). Designing large diameter, closely coupled 2-row tapered roller bearings for supporting wind turbine rotor loading, USA, The Timken Company, USA.

Lundberg, G., \& Palmgren, A. (1947). Dynamics capacity of rolling bearings. Acta Polytechnica Mechanical Engineering Series, 1(3), Stockholm, Sweden.

Niu, L. K., Yang, J. M., \& Gao, J. Y. (2013). Study on contact fatigue damage of hardened layer of yaw bearing raceway in wind turbine. Acta Energiae Solaris Sinica, 34(8), 1415-1420.

World Wind Energy Association. (2012). 2012 Half-year Report, WWEA Head Office, Bonn, Germany.

\section{Copyrights}

Copyright for this article is retained by the author(s), with first publication rights granted to the journal.

This is an open-access article distributed under the terms and conditions of the Creative Commons Attribution license (http://creativecommons.org/licenses/by/3.0/). 